\title{
Band Gaps of SH Wave Propagating Through Elastic/PE/PM Phonon Crystal
}

\author{
Huijuan Zhou and Man Lan \\ Department of mathematics and science, Luoyang Institute of Science and \\ Technology, Luoyang, 471023, China. \\ lyzhouhuijuan@163.com
}

\begin{abstract}
Dispersive characteristics of SH waves propagating with an angle $\theta$ through onedimensional elastic/piezoelectric/piezomagnetic phononic crystal are studied in this paper. Firstly, Secondary horizontal wave (SH wave) are studied in`this paper. Secondly, the transfer matrix method is used to derive the dispersion equations. The solving process is as follow. The state vectors on both sides of the sub-ldyer in a unit cell are connected by a matrix, and those on both sides of the interface between the adjacent sub-layers are connected by an identical matrix. Thus, a matrix connecting the state vectors of both sides of the unit cell can be gotten, and the dispersion equationduill be derived with the Bloch Theory. Finally, the effect of the componen ratio is considered.
\end{abstract}

Keywords: SH wave; transfer matrix method, component ratio; phononic crystal

\section{Introduction}

Phononic crystal (PC) Ba sort of aftificial periodicalstructure composed of different materials. The study of phrnoni crystals has attracted wide attention in the past decades [1-5] because it gives birth to complete acoustic band gaps within which elastic waves can't propagate hile in other frequencies range they can transmit without amplitude fading. PC has a broad application prospect in many fields. For example, PCs can be used to control the propagation ofelastic waves due to the presence of band gaps [6,7], such as vibration isolation and noise reduction.

Recently, the research on PCs have evolved into a new trend, that is, from traditional phononic crystals which are made by elastic materials to phononic crystals made by piezoelectric(PE) and piezomagnetic (PM). There are lots of reseaches about piezoelectric PCs. Rodriguez-Ramos et al. [8] applied the global matrix method to find the behavior of transmission coefficients for shear horizontal wave propagation with oblique incidence in piezocomposite layered systems. Degraeve et al. [9] studied the width of stop bands in a superlattice made of alternate rods constituted of piezoelectric ceramic and elasic materialWang et al. [10] studied the stop band properties of elastic waves in threedimensional piezoelectric phononic crystals with initial stress by taking the mechanical and electrical coupling into account. Guo and Wei [11] studied the effects of mechanically and dielectrically imperfect interfaces on dispersion relations of elastic waves in a onedimensional piezoelectric phonoic crystal. Bian et al. [12] investigated band gaps are tuned as the temperature changed. There are also some researches on piezoelectric/piezomagnetic PCs. Pang et al. [13] studied dispersive behavior and band structure of $\mathrm{SH}$ waves in magnetic-electric periodically layered plate by the transfer matrix method. Sun et al. [14] assumed that the interface in the composite is damaged mechanically, magnetically or electrically, and then obtained for two kinds of electricmegnetic boundary conditions at the free surface. Kuo and Chen [15] studied the effective magnetoelectricity of piezoelectric-piezomagnetic fibrous composites with imperfect 
interfaces subjected to anti-plane shear deformation coupled to in-plane electromagnetic fields. Guo and Wei [16] studied the dispersion relations of elastic waves in a onedimensional phononic crystal formed by periodically repeating of a pre-stressed piezoelectric slab and a pre-stressed piezomagnetic slab. Lan and Wei [17] studied the band gaps of a laminated piezoelectric/piezomagnetic phononic crystal with graded interlayer. Most of these papers are about PCs formed with two phases.

However, PCs with more than two phases are necessary in life to satisfy different requirements. Wang et al. [18] investigated the elastic wave propagation in phononic crystals with piezoelectric and piezomagnetic inclusions taking the magnetic-electroelastic coupling into account. Giordano [19] proposed an explicit procedure for the homogenization of laminated magneto-electro-elastic nonlinear materials to determine the effective response of a multilayered structure composed of materials with an arbitrarily nonlinear and anisotropic coupled behavior. Piliposyan [20] investigated the existence and propagation of a surface SH wave at the interface of two-magneto-electro-elastic halfspaces.

All these papers focused on PCs made up by two materials. In this paper, PC made up by three different materials (elastic/PE/PM) will be studied.

\section{Constitutive and Governing Equations for Elastic/PE/PM PC}

The one-dimensional (1-D) elastic/PE/PMPeriodic laminated structure consists of elastic layers, piezoelectric layers and piezomagnetic 1ayers alternately. The local orthogonal Cartesian coordinates system, namely $\left(x_{k}, y, z_{k}\right)$, are established with the origin at the left boundary of each sub-layef. $z$ axis is perpendicular to the planar interface in K-th unit cell. This model system is 1lustrated in Figure 1.

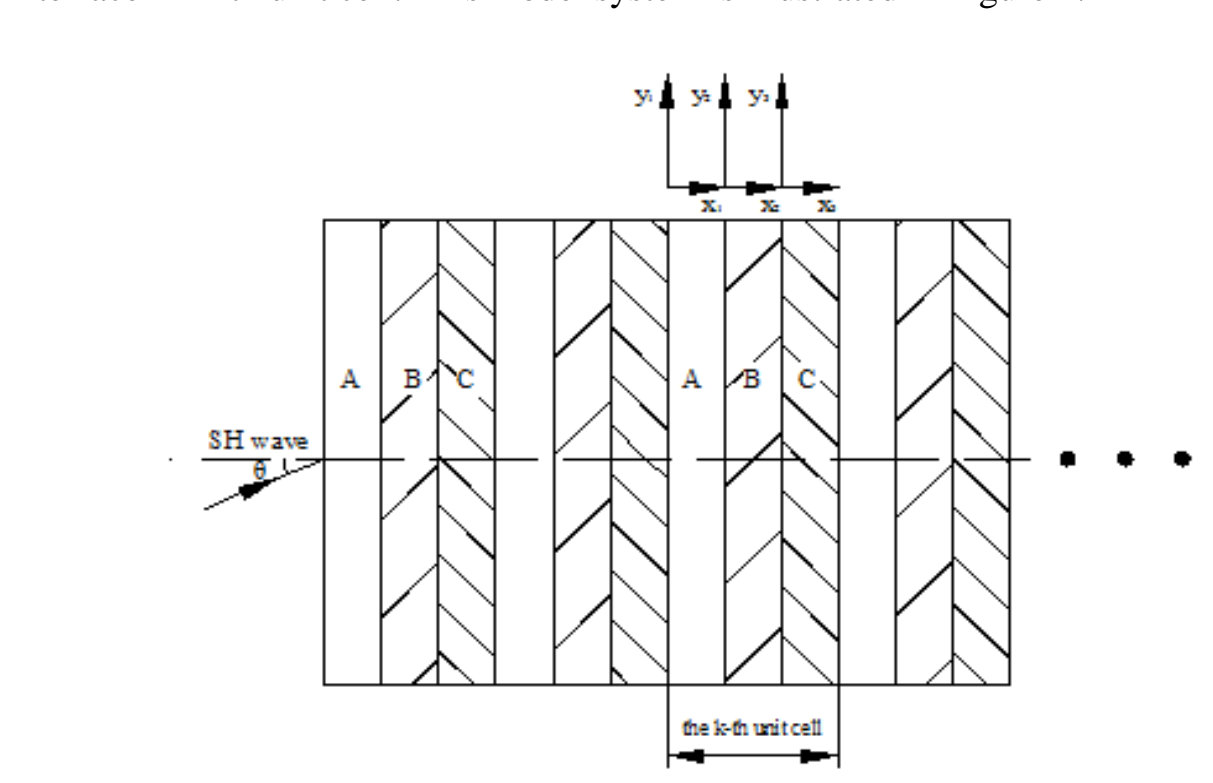

Figure 1. Schematic Illustration of One 1D Elastic/Piezoelectric PC

The unite cell consists of three materials, elastic material, piezoelectric matrial (PE) and piezomagenatic material (PM). The width in the $x_{2}$-direction is much larger than the lattice length, so it can be seemed as an infinite length. $d_{A}, d_{B}$ and $d_{C}$ are the widths of the elastic, PE and PM phases, respectively. Therefore, the lattice length are given by

$$
d=d_{A}+d_{B}+d_{C}
$$

For the layered magneto-electro-elastic solid, the fully coupled constitutive relation can be written as 


$$
\left[\begin{array}{l}
\sigma \\
D \\
B
\end{array}\right]=\left[\begin{array}{ccc}
C & -e^{T} & -q^{T} \\
e & \varepsilon & \alpha \\
q & \alpha^{T} & \mu
\end{array}\right]\left[\begin{array}{l}
S \\
E \\
H
\end{array}\right]
$$

where $\boldsymbol{\sigma}, \boldsymbol{D}$ and $\boldsymbol{B}$ are the stress tensor, electric displacement and magnetic induction, respectively; $\boldsymbol{C}, \boldsymbol{\varepsilon}$ and $\boldsymbol{\mu}$ are the stiffness tensor, dielectric and magnetic permeability tensor, respectively; $\boldsymbol{e}, \boldsymbol{q}$ and $\boldsymbol{\alpha}$ are the PE, PM and meganeto-electrico coefficient tensors, respectively; $\boldsymbol{s}, \boldsymbol{E}$ and $\boldsymbol{H}$ are the strain tensor, electrical field and magnetic field, respectively. The constitutive relation of PE phase or PM phase will be respectively getten by taking $\boldsymbol{q}=\boldsymbol{\alpha}=\mathbf{0}$ or $\boldsymbol{e}=\boldsymbol{\alpha}=\mathbf{0}$.

In this paper, the transversely isotropic PE and PM materials are considered. The stiffness tensor, PE coefficient and PM coefficient can be expressed as

$$
\begin{aligned}
& \boldsymbol{C}=\left[\begin{array}{llllll}
c_{11} & c_{12} & c_{13} & & & \\
c_{12} & c_{11} & c_{13} & & & \\
c_{13} & c_{13} & c_{33} & & & \\
& & & c_{44} & & \\
& & & & c_{44} & \\
& & & & & c_{44}
\end{array}\right], \boldsymbol{e}=\left[\begin{array}{r}
e_{31} \\
e_{31} \\
\\
e_{33}
\end{array}\right] \boldsymbol{q}=\left[\begin{array}{l}
q_{31} \\
q_{31} \\
q_{33} \\
e_{15}
\end{array}\right], \\
& \boldsymbol{\varepsilon}=\left[\begin{array}{llll}
\varepsilon_{11} & & \\
& \varepsilon_{11} & \\
& & \varepsilon_{33}
\end{array}\right], \boldsymbol{\mu}=\left[\begin{array}{lll}
\mu_{11} & & \\
& \mu_{11} & \\
& & \mu_{33}
\end{array}\right],
\end{aligned}
$$

where other elements equal to zero.

The strain-displacement relation the equation with the electric field $\boldsymbol{E}$ and an electric potential $\varphi$ and the equation with the magnetic field $\boldsymbol{H}$ and a meganetic potential $\phi$ are given, respectively,

$$
S_{i, j}=0.5\left(u_{i, j}+u_{j, i}\right), E_{i}=-\varphi_{, i}, H_{i}=-\phi_{, i}
$$

When SH-wave propagating through the structure with an angle $\theta(0<\theta<\pi / 2)$ the displacements in $x_{1} \rightarrow x_{3}$ directions and the magnetic potential can be written as

$u=0, v=0, w=w(x, y, t), \varphi=\varphi(x, y, t), \phi=\phi(x, y, t)$

By substituting Eqs. (4) and (5) into Eq. (2), the governing equtaions for the elastic , $\mathrm{PE}$ and PM medium can be respectively obtained as,

$$
\begin{gathered}
c_{44}^{A} \nabla^{Z} w_{1}=\rho^{B} \ddot{w}_{1},-\varepsilon_{11}^{A} \nabla^{2} \varphi_{1}=0 \\
c_{44}^{B} \nabla^{2} w_{2}+e_{15}^{B} \nabla^{2} \varphi_{2}=\rho^{B} \ddot{w}_{2}, e_{15}^{B} \nabla^{2} w_{2}-\varepsilon_{11}^{B} \nabla^{2} \varphi_{2}=0 \\
e_{44}^{C} \nabla^{2} w_{2}+q_{15}^{C} \nabla^{2} \phi_{3}=\rho^{C} \ddot{w}_{3}, q_{15}^{C} \nabla^{2} w_{2}-\mu_{11}^{C} \nabla^{2} \phi_{3}=0
\end{gathered}
$$

Where the superscripts A, B and C denote the elastic, PE and PM material, respectively.

For the present crystals, the solution to Eqs. (6) (8) have the form

$$
\boldsymbol{u}=\operatorname{Uexp}\left(i k_{y} y-i \omega t\right)
$$

where $\boldsymbol{u}=\{w, \varphi, \phi\}$ are the displacement, electric potential and magnetic potential; and $\mathbf{U}=\{\mathrm{W}, \Psi, \Phi\}$ are the amplitudes of the displacement, electric potential and magnetic potential; $\omega$ is the angular frequency; $k_{y}=k \sin \theta, k$ is the Bloch wave vector and $\theta$ is the propagating angle. Inserting Eq. (9) into Eqs. (6) (8) yields

$$
\begin{gathered}
w(x, y, t)=\left[A e^{-i r x}+B e^{i r x}\right] \exp \left(i k_{y} y-i \omega t\right) \\
\varphi(x, y, t)=\left[C e^{-k_{y} x}+D e^{k_{y} x}+p_{1}\left(A e^{-i r x}+B e^{-i r x}\right)\right] \exp \left(i k_{y} y-i \omega t\right) \\
\phi(x, y, t)=\left[E e^{-k_{y} x}+F e^{k_{y} x}+p_{2}\left(A e^{-i r x}+B e^{-i r x}\right)\right] \exp \left(i k_{y} y-i \omega t\right)
\end{gathered}
$$


where $A, B, C, D, E$ and $F$ are undifined coefficients; $r=\sqrt{k_{t}^{2}-k_{y}^{2}}, k_{t}=\omega / c_{t}$, $c_{t}=\sqrt{\hat{c}_{44} / \rho} \quad, \quad \hat{c}_{44}=c_{44}+\hat{p} \quad, \quad \hat{p}=p_{1} e_{15}+p_{2} q_{15}$ $p_{1}=\left(q_{15} \alpha_{11}-e_{15} \mu_{11}\right) /\left(\alpha_{11}^{2}-\varepsilon_{11} \mu_{11}\right), p_{2}=\left(e_{15} \alpha_{11}-q_{15} \varepsilon_{11}\right) /\left(\alpha_{11}^{2}-\varepsilon_{11} \mu_{11}\right)$.

Then the corresponding stress, electric dis-placement and magnetic induction component will be obtained by inserting Eqs. (10) (12) into Eq. (2),

$$
\begin{aligned}
& \sigma_{z x}(x, y, t)=\left\{i r\left(c_{44}+p_{1} e_{15}+p_{2} q_{15}\right)\left(-A e^{-i r x}+B e^{i r x}\right)+e_{15} k_{y}\left(-C e^{-k_{y} x}+\right.\right. \\
& \left.\left.D e^{k_{y} x}\right)+\quad q_{15} k_{y}\left(-E e^{-k_{y} x}+F e^{k_{y} x}\right)\right\} e^{i k_{y} y-i \omega t} \text {, } \\
& D_{x}(x, y, t)=\left\{i r\left(e_{15}-\varepsilon_{11} p_{1}-\alpha_{11} p_{2}\right)\left(-A e^{-i r x}+B e^{i r x}\right)-\varepsilon_{11} k_{y}\left(-C e^{-k_{y} x}+\right.\right. \\
& \left.\left.D e^{k_{y} x}\right)-\quad \alpha_{11} k_{y}\left(-E e^{-k_{y} x}+F e^{k_{y} x}\right)\right\} e^{i k_{y} y-i \omega t}, \\
& B_{x}(x, y, t)=\left\{\operatorname{ir}\left(q_{15}-\alpha_{11} p_{1}-\mu_{11} p_{2}\right)\left(-A e^{-i r x}+B e^{i r x}\right)-\alpha_{11} k_{y}\left(-C e^{-k_{y} x}+\right.\right. \\
& \left.\left.D e^{k_{y} x}\right)-\quad \mu_{11} k_{y}\left(-E e^{-k_{y} x}+F e^{k_{y} x}\right)\right\} e^{i k_{y} y} j^{i \omega t} .
\end{aligned}
$$

By setting a state vector $\mathbf{V}(x, y, t)=\left(\mathrm{w}, \sigma_{z x}, \varphi, D_{x}, \phi B_{x}\right)^{3}$, the pysical relationship of the left and right side in a phase can be expressed as

$$
\mathbf{V}\left(d_{j}\right)=\mathbf{T}_{\mathbf{j}} \mathbf{V}(0)
$$

where the subscript $j=A, B, C$ denotes different phasein a unit cell. Then for a unit cell consisting of an elastic phase, a PE phase and a PM phase, the total transfer matrix of the unit cell will be derived as

$$
\mathbf{V}(d)=\mathbf{T V}(0), \boldsymbol{T}=\mathbf{T}_{\mathrm{C}} \mathbf{T}_{\mathrm{B}} \mathbf{T}_{\mathrm{A}},
$$

where $\mathbf{T}_{\mathrm{j}}(j=A, B, C)$ which can be derivedffrom Eq. (16) are listed as follow,

$$
\begin{aligned}
& T_{11}=\cos \left(d_{j} r_{j}\right), T_{12}=\frac{Q}{\sqrt{3\left(\hat{c}_{44}+Q\right)}} \sin \left(\hat{d}_{j} r_{j}, T_{13}=0, T_{14}=\frac{p_{1}}{r_{j}\left(\hat{c}_{44}+Q\right)} \sin \left(d_{j} r_{j}\right), T_{15}=0\right. \\
& T_{16}=p_{2} \sin \left(d_{j} r_{j}\right) /\left[x_{i}\left(\hat{c}_{44}+Q\right)\right]_{,} T_{21}=-r_{j} \hat{c}_{44} \sin \left(d_{j} r_{j}\right)-\frac{1}{2} \hat{p} k_{y}\left(e^{d_{j} k_{y}}-e^{-d_{j} k_{y}}\right) \text {, } \\
& T_{22}=\left[\hat{c}_{44} \cos \left(\hat{d}_{j} r_{i}\right)+\frac{1}{2} Q\left(e^{d j k_{y}}+e^{-d_{j} k_{y}}\right)\right] /\left(\hat{c}_{44}+Q\right) \quad, \quad T_{23}=\frac{1}{2} e_{15} k_{y}\left(e^{d_{j} k_{y}}-\right. \\
& \left.e^{-d_{j} k_{y}}\right) \text {, } \\
& T_{24}=\frac{p_{1} \hat{c}_{44}}{\left(\hat{c}_{44}+Q\right)}\left[\cos \left(d_{j} \gamma_{j}\right)-\frac{1}{2}\left(e^{d_{j} k_{y}}+e^{-d_{j} k_{y}}\right)\right], T_{25}=\frac{1}{2} q_{15} k_{y}\left(e^{d_{j} k_{y}}-e^{-d_{j} k_{y}}\right), \\
& T_{26}=\frac{p_{2} \hat{c}_{44}}{\left(\hat{c}_{44}+Q\right)}\left[\cos \left(d_{j} r_{j}\right)-\frac{1}{2}\left(e^{d_{j} k_{y}}+e^{-d_{j} k_{y}}\right)\right] \quad, \quad T_{31}=p_{1}\left[\cos \left(d_{j} r_{j}\right)-\frac{1}{2}\left(e^{d_{j} k_{y}}+\right.\right. \\
& \left.\left.e^{-d_{j} k_{y}}\right)\right] \text {, } \\
& T_{32}=\frac{1}{\hat{\varepsilon}_{44}}\left[\frac{p_{Y}}{r_{j}} \sin \left(d_{j} r_{j}\right)+\frac{\left(\alpha_{11} N-\mu_{11} M\right)}{2 k_{y}\left(\alpha_{11}^{2}-\varepsilon_{11} \mu_{11}\right)}\left(e^{d_{j} k_{y}}-e^{-d_{j} k_{y}}\right)\right], T_{33}=\frac{1}{2}\left(e^{d_{j} k_{y}}+e^{-d_{j} k_{y}}\right) \text {, } \\
& T_{34}=\frac{p_{1}^{2}}{r_{j}\left(\hat{c}_{44}+Q\right)} \sin \left(d_{j} r_{j}\right)+\frac{1}{2 k_{y}\left(\alpha_{11}^{2}-\varepsilon_{11} \mu_{11}\right)}\left[\frac{p_{1}}{\hat{c}_{44}+Q}\left(\alpha_{11} N-\mu_{11} M\right)+\mu_{11}\right]\left(e^{d_{j} k_{y}}-\right. \\
& \left.e^{-d_{j} k_{y}}\right) \quad, \quad T_{36}=\frac{p_{1} p_{2}}{r_{j}\left(\hat{c}_{44}+Q\right)} \sin \left(d_{j} r_{j}\right)+\frac{1}{2 k_{y}\left(\alpha_{11}^{2}-\varepsilon_{11} \mu_{11}\right)}\left[\frac{p_{2}}{\hat{c}_{44}+Q}\left(\alpha_{11} N-\mu_{11} M\right)-\right. \\
& \left.\alpha_{11}\right]\left(e^{d_{j} k_{y}}-e^{-d_{j} k_{y}}\right), \quad T_{35}=0, T_{41}=-r_{j} M \sin \left(d_{j} r_{j}\right)+\frac{k_{y}}{2}\left(p_{1} \varepsilon_{11}+p_{2} \alpha_{11}\right)\left(e^{d_{j} k_{y}}-\right. \\
& \left.e^{-d_{j} k_{y}}\right) \text {, } \\
& T_{42}=\frac{M}{c_{44}+Q}\left[\cos \left(d_{j} r_{j}\right)-\frac{1}{2}\left(e^{d_{j} k_{y}}+e^{-d_{j} k_{y}}\right)\right], T_{43}=-\frac{1}{2} \varepsilon_{11} k_{y}\left(e^{d_{j} k_{y}}-e^{-d_{j} k_{y}}\right), \\
& T_{44}=\frac{p_{1} M}{\hat{c}_{44}+Q} \cos \left(d_{j} r_{j}\right)+\frac{1}{2}\left(1-\frac{p_{1} M}{\hat{c}_{44}+Q}\right)\left(e^{d_{j} k_{y}}+e^{-d_{j} k_{y}}\right), \quad T_{45}=-\frac{1}{2} \alpha_{11} k_{y}\left(e^{d_{j} k_{y}}-\right. \\
& \left.e^{-d_{j} k_{y}}\right) \text {, } \\
& T_{46}=\frac{p_{2} M}{\hat{c}_{44}+Q}\left[\cos \left(d_{j} r_{j}\right)-\frac{1}{2}\left(e^{d_{j} k_{y}}+e^{-d_{j} k_{y}}\right)\right], T_{51}=p_{2}\left[\cos \left(d_{j} r_{j}\right)-\frac{1}{2}\left(e^{d_{j} k_{y}}+e^{-d_{j} k_{y}}\right)\right], \\
& T_{52}=\frac{1}{\hat{c}_{44}+Q}\left[\frac{p_{2}}{r_{j}} \sin \left(d_{j} r_{j}\right)+\frac{M \alpha_{11}-N \varepsilon_{11}}{2 k_{y}\left(\alpha_{11}^{2}-\varepsilon_{11} \mu_{11}\right)}\left(e^{d_{j} k_{y}}-e^{-d_{j} k_{y}}\right)\right], T_{53}=0,
\end{aligned}
$$




$$
\begin{aligned}
& T_{54}=\frac{p_{1} p_{2}}{r_{j}\left(\hat{c}_{44}+Q\right)} \sin \left(d_{j} r_{j}\right)+\frac{\left(e^{d_{j} k_{y}}-e^{-d_{j} k_{y}}\right)}{2 k_{y}\left(\alpha_{11}^{2}-\varepsilon_{11} \mu_{11}\right)}\left[\frac{p_{1}\left(M \alpha_{11}-N \varepsilon_{11}\right)}{\hat{c}_{44}+Q}-\alpha_{11}\right], \\
& T_{55}=\frac{1}{2}\left(e^{d_{j} k_{y}}+e^{-d_{j} k_{y}}\right), \\
& T_{56}=\frac{p_{2}^{2}}{r_{j}\left(\hat{c}_{44}+Q\right)} \sin \left(d_{j} r_{j}\right)+\frac{\left(e^{d_{j} k_{y}}-e^{-d_{j} k_{y}}\right)}{2 k_{y}\left(\alpha_{11}^{2}-\varepsilon_{11} \mu_{11}\right)}\left[\frac{p_{1}\left(M \alpha_{11}-N \varepsilon_{11}\right)}{\hat{c}_{44}+Q}-\alpha_{11}\right], \\
& T_{61}=-r_{j} N \sin \left(d_{j} r_{j}\right)+\frac{k_{y}}{2}\left(p_{1} \alpha_{11}+p_{2} \mu_{11}\right)\left(e^{d_{j} k_{y}}-e^{-d_{j} k_{y}}\right), \\
& T_{62}=\frac{N}{\hat{c}_{44}+Q}\left[\cos \left(d_{j} r_{j}\right)-\frac{1}{2}\left(e^{d_{j} k_{y}}+e^{-d_{j} k_{y}}\right)\right], T_{63}=-\frac{1}{2} \alpha_{11} k_{y}\left(e^{d_{j} k_{y}}-e^{-d_{j} k_{y}}\right), \\
& T_{64}=\frac{p_{1} N}{\hat{c}_{44}+Q}\left[\cos \left(d_{j} r_{j}\right)-\frac{1}{2}\left(e^{d_{j} k_{y}}+e^{-d_{j} k_{y}}\right)\right], T_{65}=-\frac{1}{2} \mu_{11} k_{y}\left(e^{d_{j} k_{y}}-e^{-d_{j} k_{y}}\right), \\
& T_{44}=\frac{p_{2} N}{\hat{c}_{44}+Q} \cos \left(d_{j} r_{j}\right)+\frac{1}{2}\left(1-\frac{p_{2} M}{\hat{c}_{44}+Q}\right)\left(e^{d_{j} k_{y}}+e^{-d_{j} k_{y}}\right), \\
& \text { where } Q=p_{1} M+p_{2} N, M=e_{15}-\varepsilon_{11} p_{1}-\alpha_{11} p_{2}, M=q_{15}-\alpha_{11} p_{1}-\mu_{1} p_{2}
\end{aligned}
$$

Meanwhile, the state vectors in the left and right side of the unit celvsatisfy the Bloch theorem in periodic structure,

$$
\mathbf{V}(d)=e^{i k_{x} d} \mathbf{V}(0) \text {. }
$$

Then, from Eq. (17) and Eq. (18), we will get that

$$
\left(\mathbf{T}-\mathbf{I} e^{i k_{x} d}\right) \mathbf{V}(0)=\mathbf{0}
$$

The non-trivial solution of Eq. (19) Crequires the crefficient determinant equal to zero, that is,

$\left|\mathbf{T}-\mathbf{I} e^{i k_{x} d}\right|=f\left(\omega, k_{x}, k_{y}\right) \leq 0$

which is the dispersive relation of the $\mathrm{SH}_{\mathrm{H}}$ wave propagating through the structure. From Eq. (20) the "pass bands" and "stop bands" will be gotten.

\section{Example and Numerical Results}

In this example, the elastic, PE and PM material are polythene, PZT-4 and $\mathrm{CoFe}_{2} \mathrm{O}_{4}$, respectively. We wilhmainly talk about the component ratio effect on band gaps. For a unit cell, the component ratio $d_{A}: d_{B}: d_{C}$ change from $2: 1: 1$ to $1: 2: 1$, and then to $1: 1: 2$. The material coefficents are given in Table 1 .

Table 1. Material Constants of Polythene, PZT-4 and $\mathrm{CoFe}_{2} \mathrm{O}_{4}[21]$

\begin{tabular}{llll} 
Material constants & Polythene & PZT-4 & $\mathrm{CoFe}_{2} \mathrm{O}_{4}$ \\
\hline$\rho\left(\mathrm{kg} / \mathrm{m}^{3}\right)$ & 1780 & 7500 & 2300 \\
$c_{11}\left(\times 10^{9} \mathrm{~N} / \mathrm{m}^{2}\right)$ & - & 132 & 286 \\
$c_{33}\left(\times 10^{9} \mathrm{~N} / \mathrm{m}^{2}\right)$ & 12 & 115 & 269.5 \\
$c_{44}\left(\times 10^{9} \mathrm{~N} / \mathrm{m}^{2}\right)$ & 7 & 26 & 45.3 \\
$c_{13}\left(\times 10^{9} \mathrm{~N} / \mathrm{m}^{2}\right)$ & - & 73 & 170.5 \\
$e_{15}\left(\mathrm{C} / \mathrm{m}^{2}\right)$ & 0 & 10.5 & 0 \\
$e_{33}\left(\mathrm{C} / \mathrm{m}^{2}\right)$ & -0.027 & 14.1 & 0 \\
$e_{31}\left(\mathrm{C} / \mathrm{m}^{2}\right)$ & 0 & -4.1 & 0 \\
$q_{15}(\mathrm{~N} / \mathrm{Am})$ & 0 & 0 & 550 \\
$q_{31}(\mathrm{~N} / \mathrm{Am})$ & 0 & 0 & 580.3 \\
$q_{33}(\mathrm{~N} / \mathrm{Am})$ & 0 & 0 & 699.7 \\
$\varepsilon_{11}\left(\times 10^{-9} \mathrm{C}^{2} / \mathrm{Nm}^{2}\right)$ & 0.2036 & 7.1 & 0.08 \\
$\varepsilon_{33}\left(\times 10^{-9} \mathrm{C}^{2} / \mathrm{Nm}^{2}\right)$ & 0.6726 & 5.8 & 0.093
\end{tabular}




$$
\begin{array}{llll}
\mu_{11}\left(\times 10^{-6} \mathrm{Ns}^{2} / \mathrm{C}^{2}\right) & 1.26 & 5.0 & 157 \\
\mu_{33}\left(\times 10^{-6} \mathrm{Ns}^{2} / \mathrm{C}^{2}\right) & 1.26 & 10.0 & 157 \\
\hline
\end{array}
$$

In Figure 2, the component ratio is 2:1:1. The elastic material is the major part in the structure. $k_{x n d}, k_{y n d}$ and $\omega_{\text {nd }}$ are respectively dimensionless $\mathrm{x}$ and y label where $k_{x}=k_{x n d} \pi / \mathrm{d}, k_{y}=k_{y n d} \pi / \mathrm{d}$ and $\omega=\omega_{\text {nd }} 2 \pi \mathrm{c}_{\mathrm{T}}^{\mathrm{B}} / \mathrm{d}$.
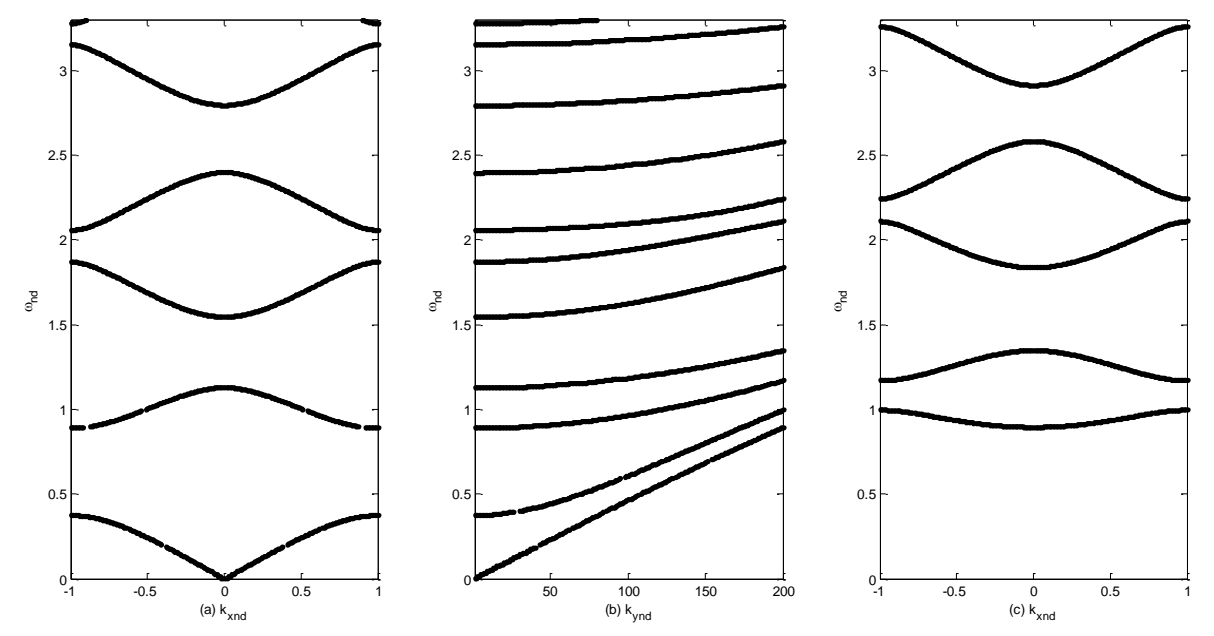

Figure 2. SH wave Propagating in Elastic/PE/PM Phononi with Component Ratio 2:1:1 (a) $k_{y}=0$; (b) $k_{y} \neq 0$; (c) $k_{y}=200$

The Figure 2(b) describes the dispersive eures when non-dimensional $k_{y}$ change from 0 to 200 , and we can observe that the change of band gaps in it. The larger the value of $k_{y}$ is, the sharper the transfer angle is. The Figure 2(a) shows the SH wave propagating perpendicular to the interface, and 2(c) shows it propagating with a certain angle $k_{y}=$ 200.
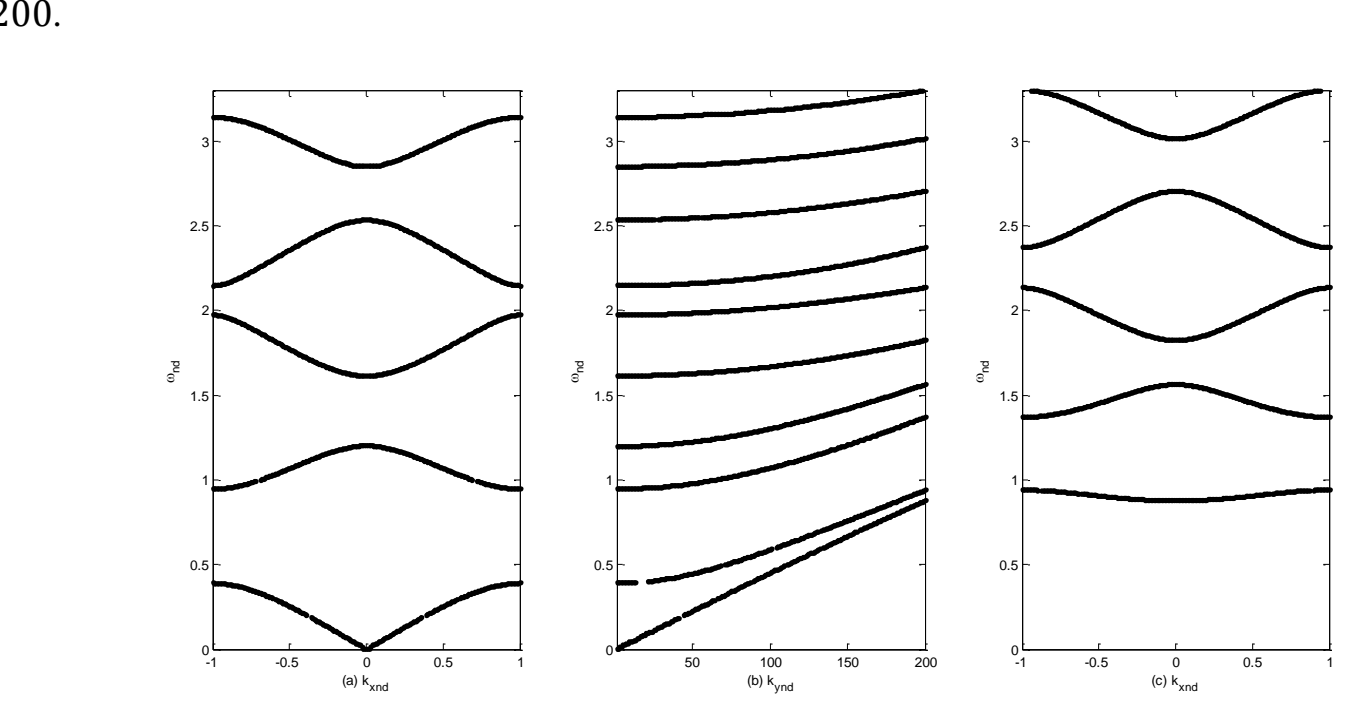

Figure 3. SH Wave Propagating in Elastic/PE/PM Phononic with Component Ratio 1:2:1(a) $k_{y}=0 ;(B) k_{y} \neq 0 ;(C) k_{y}=200$

In Figure 3, the component ratio is 1:2:1. The piezoelectric material is the major part in the structure. $k_{x n d}, k_{y n d}$ and $\omega_{\text {nd }}$ are still dimensionless $\mathrm{x}$ and $\mathrm{y}$ label, respectively. Compared with Figure 2, it can be found that the first band gap get narrower in both two 
figures, but the change in Figure 2 is much sharper than in Figure 3. In Figure 2, the second band gap turn to bigger, while that in Figure 3 turn to smaller.
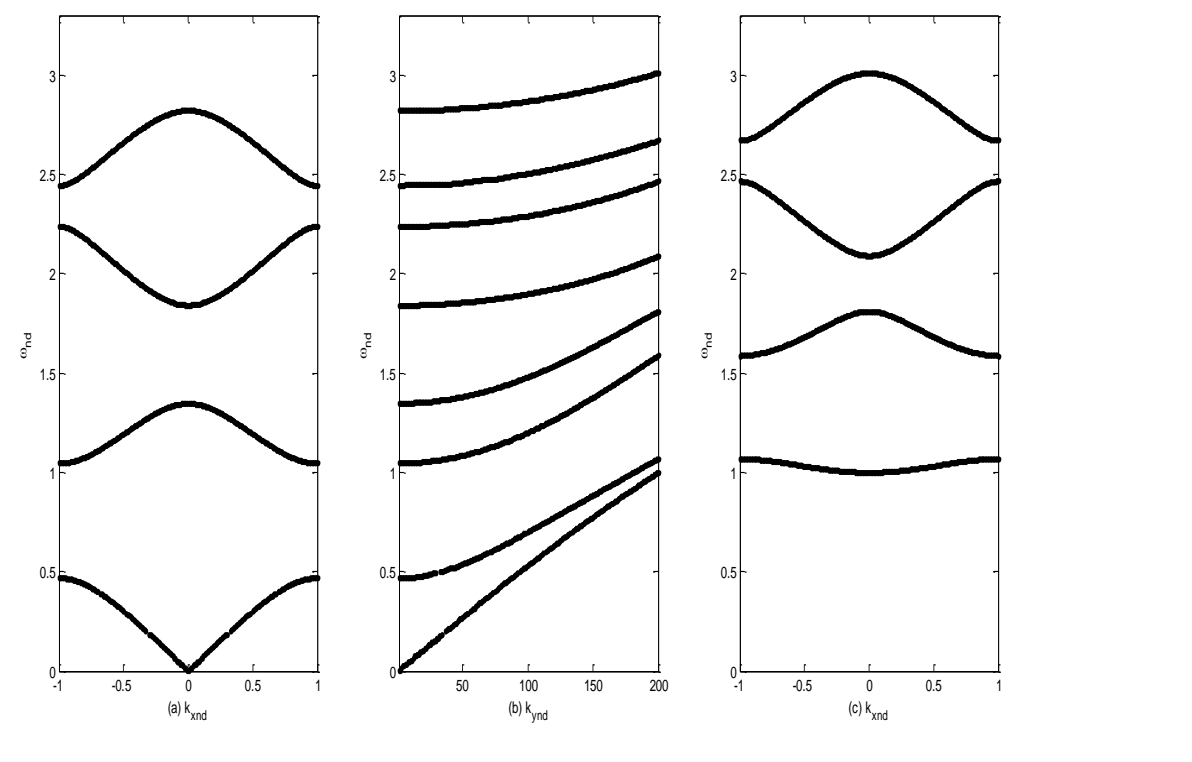

Figure 4. SH Wave Propagating in Elastic/PE/PM Phononic with Component Ratio 1:1:2(a) $\mathbf{k}_{\mathrm{y}}=9 ;(B) \mathbf{k}_{\mathrm{y}} \neq 0$; (C) $\mathbf{k}_{\mathbf{y}}=200$

There are fewer frequency curves in Figure 4 than figures 2 and 3. Hence, in this numerical example PM phase makes more effect on the dispersive curves and band gaps. Observing three figures and material coefficients, it can be found that the stiffness coefficients of PM material are much bigger than those of elastic and PE materials, while the density of PM material isn't the biggest one. So it's a proof that stiffness coefficients have more effect on band gaps thanthe density.

\section{Conclusion}

The influences of component ratio on dispersive curves in 1D elastic/PE/PM periodic structure are invesigared in this paper. The normal and oblique propagating angles are considered. It can be found that: The influences can be observed obviously when the component ratio $d_{A}: d_{B}: d_{C}$ changes from $2: 1: 1$ to $1: 2: 1$, and furthermore to $1: 1: 2$. The stiffness coefficients have more effect on band gaps than the density. With the increasing of the values of stiffness coefficients, most of the dispersive cures turn to higher frequency, but some of them still turn to lower frequency. The first and second band gaps are noticeably affected, but the tendencies are very different in the figures $2 \sim 4$. For example, the width of the first band gap turn to smaller in figure2, to smoothly smaller in Figure 3, but almost be not changed in Figure 4. The width of the second band gap turns to larger in figures 3 and 4, but to smaller in Figure 2. According to these results we can adjust the position og band gaps to satisfy different requirements in production.

\section{References}

[1] Przemyslaw Nowak and MaciejKrawczyk, "Phononic Band Gaps in One-dimensional Phononic Crystals with Nanoscale Periodic Corrugations at Interfaces.FDTD and PWM Simulations", Computational Methods in Science and Technology, vol. 16, no. 1, (2010), pp. 85-95.

[2] C. Croënne, E.J.S. Lee, Hefei Hu and J.H. Page, "Band gaps in phononic crystals: Generation mechanisms and interaction effects", AIP Advances, vol. 1, no. 4, (2011), pp. 3392-3398. 
[3] F. C. Hsu, C. I. Lee, J. C. Hsu, T. C. Huang, C. H. Wang and P. Chang, "Acoustic band gaps in phononic crystal strip waveguides”, Applied Physics Letters, vol. 96, no. 5, (2010), pp. 051902-0519023.

[4] J. T. Barreiro, "Phononic crystals: Entering an acoustic phase", Nature Physics, vol. 11, no. 3, (2015), pp. $215-216$

[5] J. C. Hsu and C. H. Hsu, "Edge waves and resonances in two-dimensional phononic crystal plates", Journal of Applied Physics, vol. 117, no. 17, (2015), pp. 056608-92.

[6] R. Jü rgen, Wook Jo, Klaus T. P. Seifert, E.-M. Anton and T. Granzow, "Perspective on the Development of Lead-free Piezoceramics", Journal of the American Ceramic Society, vol. 92, no. 6, (2009), pp. 1153-1177.

[7] S. Zhang and F. Yu,"Piezoelectric Materials for High Temperature Sensors",Journal of the American Ceramic Society, vol. 94, no. 10, (2011), pp.3153-3170.

[8] R. Rodrí guez-Ramos, H. Calás, J. A. Otero, B. Guerra, A. Ramos and Y.S. Wang. "Shear horizontal wave in multilayered piezoelectric structures: effect of frequency, incidence angle and constructiye parameters", International Journal of Solids and Structure, vol. 48, no. 20, (2011), pp. 2941-2947

[9] S. Degraeve, C. Granger, B. Dubus, J. O. Vasseur, M. Pham Thinand A. C. Hladky-Hennion, "Tunability of a one-dimensional elastic/piezoelectric phononic crystal using extental capacitances", ActaAcustica united with Acustica, vol. 101, no. 3, (2015), pp. 494-501.

[10] Y. Z. Wang, F. M. Li, K. Kishimoto, Y. S. Wang and W. H Huang, “Band gaps of elastic waves in three-dimensional piezoelectric phononic crystals with initial stress European Journal of Mechanics A/Solids, vol. 29, no. 2, (2010), pp. 182-189.

[11] X. Guo and P. J. Wei, "Dispersion relations of elastic wave in one-dimensional piezoelectric phononic crystal with mechanically and dielectrical imperfect interface", Mechanics of Materials, vol. 93, (2016), pp. 168-183.

[12] Z. Bian, W. Peng and J. Song, "Thermal tuning of band structures in a one-dimensional phononic crystal”, Journal of Applied Mechanics, vol 81 no 4, (2013), pp. $943-960$.

[13] Y. Pang, J. S.Gao, J. X. Liu. "SH wave propagation in magnetie-electric periodically layered plates", Ultrasonics, vol. 54, no. 5, (2014), pp. 1341-1349.

[14] W. H. Sun, G. L. Ju, J. W, Pan and Y. D. L, "Effects of the imperfect interface and piezoelectric/piezomagnetic stiffening on the SH wave in a multiferroic composite", Ultrasonics, vol. 51, no. 7, (2011), pp. 831-838.

[15] H. Y. Kuo and C. Y. Chen, "Decoupling transformation for piezoelectric-piezomagnetic fibrous composites with imperfect interfaces", Intenational Journal of Solids and Structures, vol 54, (2015), pp. 111-120.

[16] X. Guo and A. J. Wei, "Dispersion relations of elastic waves in one-dimensional piezoelectric/piezomagnetic phonohiclcrystal with initial stresses", Ultrasonics, vol. 66, (2016), pp. 7285.

[17] M. Lan and P. J. Wei, "Band gap of piezoelectric/piezomagnetic phononic crysta with graded interlayer', Acta Mechanico, vol. 225, no. 6, (2014), pp. 1779-1794.

[18] Y. Z. Wang, F. M. Li, W. H. Huang, X. Jiang, Y. S. Wang and K. Kishimoto,"Wave band gaps in twodimensional piezoelectric/piezomagnetic phononic crystals", International Journal of Solids and Structures, vol. 45, no 14, (2008), pp. 4203-4210.

[19] S Giordano, "Explicit nonlinear homogenization for magneto-electro-elastic laminated materials", Mechanics Research Communications, vol. 55, no. 1, (2014), pp. 18-29.

[20] D. Piliposyan, "Shear surface waves at the interface of two magneto-electro-elastic media", Multidiscipline Modeling in Materials and Structures, vol. 8, no. 3, (2012), pp. 417-426.

[21] E. Pan, "Exact solution for simply supported and multilayered magneto-electro-elatic plates", Journal of Applied Mechanics, vol. 68, no. 4, (2001), pp. 608-618.
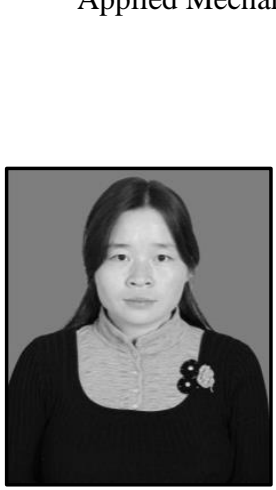

\section{Authors}

Huijuan Zhou, She was born in 1981 in Luoyang city, henan province, in 2006 graduated from xinyang normal university, Master of Science. She is a lecturer in Luoyang institute of science and technology of department of mathematics and physics. The main research interest is in semigroup of algebra. 


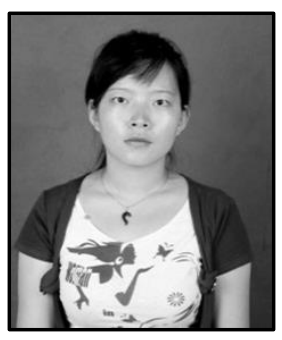

Lan Man, She was born in 1985 in Rushan city, shandong province, in 2014 graduated from university of science and technology Beijing, Doctor of Engineering. She is a lecturer in Luoyang institute of science and technology of department of mathematics and physics. The main research interest is in composite materials.

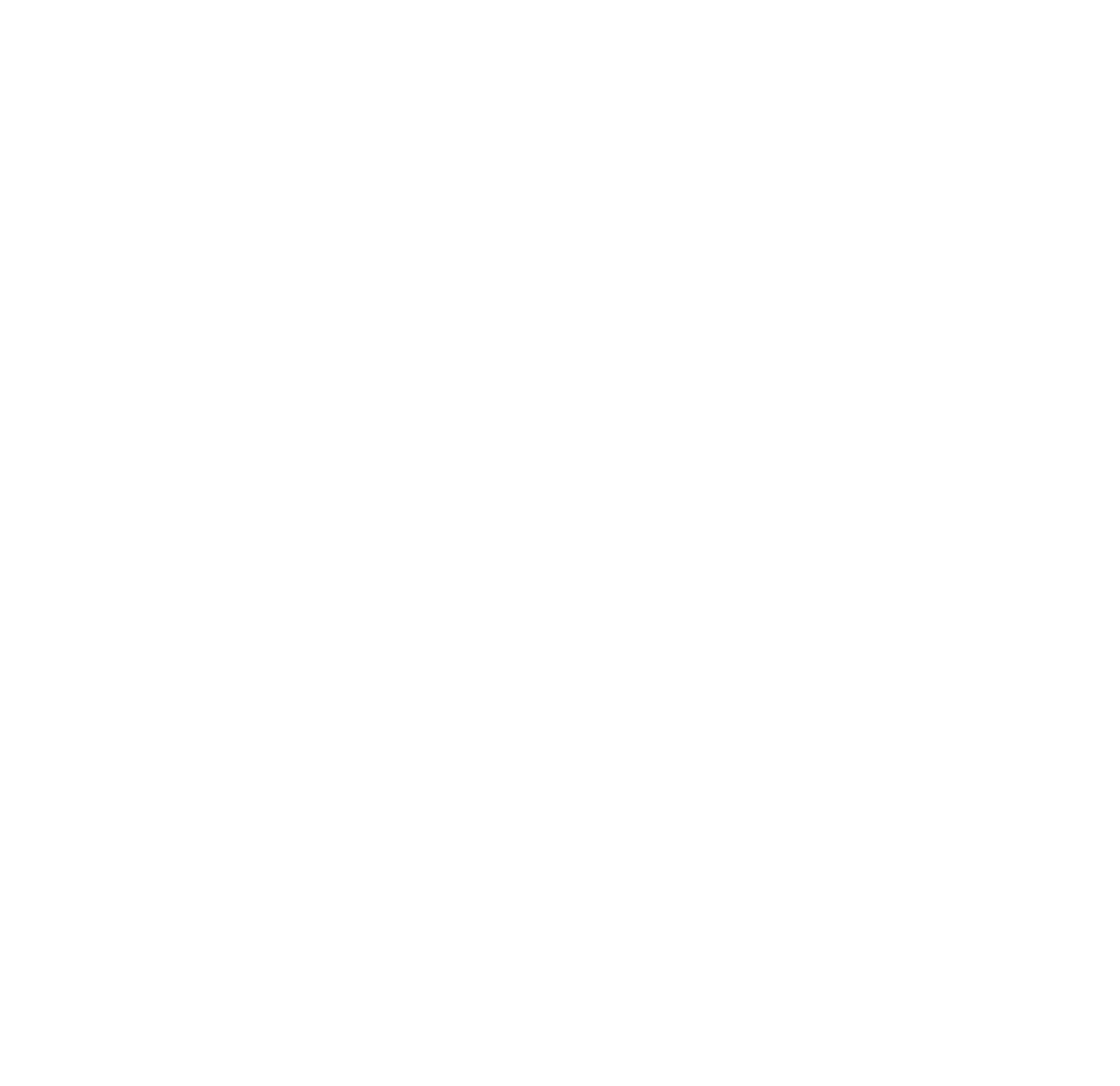


International Journal of Control and Automation

Vol. 9, No. 8 (2016)

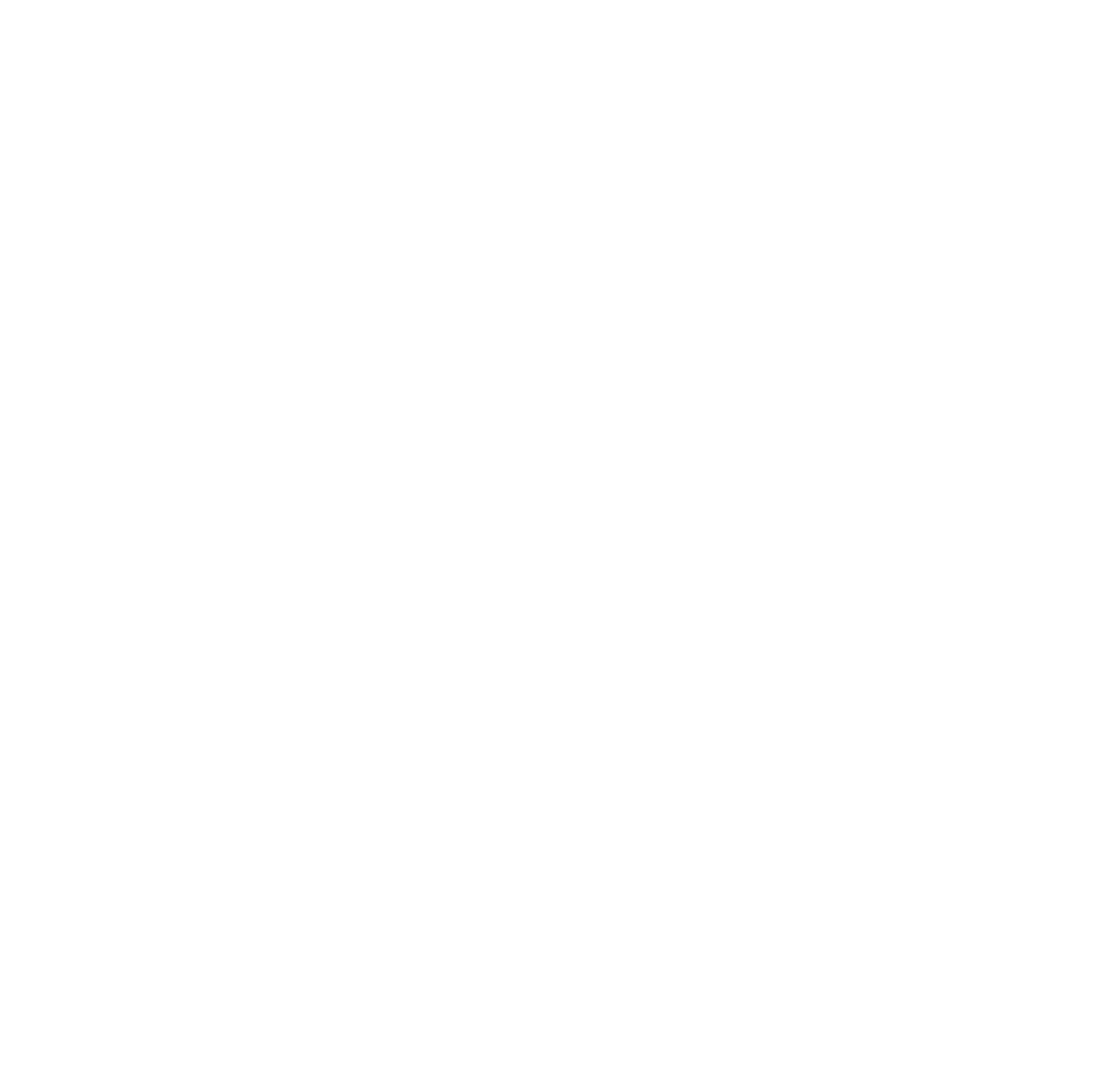

